\title{
Métodos de avaliação de densidade mineral óssea maxilomandibular por meio de tomografia Cone Beam: revisão de literatura
}

\author{
Bone mineral density assessment in maxillomandibular \\ region using Cone Beam computed tomography: a review of the literature
}

\author{
BRUNA DI PROFIO \\ BRUNA DE OLIVEIRA RECH ${ }^{1}$ \\ MARIANA MURAI CHAGAS ${ }^{1}$ \\ OTÁVIO HENRIQUE PINHATA BAPTISTA ${ }^{1}$ \\ VINICIUS TEIXEIRA SILVA ${ }^{1}$ \\ LUCIANA MUNHOZ \\ EMIKO SAITO ARITA
}

\begin{abstract}
RESUMO
Objetivo: O objetivo desta revisão bibliográfica foi descrever estudos que utilizaram métodos de análise qualitativos e quantitativos em tomografia computadorizada cone beam (TCFC) na análise da densidade mineral óssea (DMO) da região maxilomandibular. Foram incluídos nesta revisão artigos referentes ao uso da TCFC na análise da DMO da região maxilo-mandibular de 2009 até 2020, publicados nas bases de dados: PUBMED, EMBASE e Scopus. As palavras em inglês "osteoporosis", "bone mineral density" e "bone content" foram combinadas com "СBCT" e "pixel values", usando-se o operador booleano "AND"."Osteoporosis" AND "CBCT", "bone mineral density" AND "СBCT"; "bone content" AND "CBCT" AND "CBCT" AND "osteoporosis"; "pixel values" AND "CBCT" AND "bone mineral density;" pixel values" AND "CBCT" AND "bone content".

Resultados: Foram selecionados 17 artigos que investigaram o uso da TCFC no estudo da DMO maxilomandibular. Muitos pesquisadores utilizaram a microtomografia, tomografia computadorizada multislice $\mathrm{e}$ análise histomorfométrica para realizar comparações com a TCFC.

Conclusões: As evidências bibliográficas sugerem que a TCFC, tanto por meio dos seus valores de escala de cinza quanto pelos seus parâmetros 3D estruturais, se apresenta como um método de relevância clínica, confiável e fácil acesso, de análise proporcional da densidade óssea da região maxilomandibular.

Palavras-chave: Osteoporose. Densidade Óssea. Tomografia Computadorizada de Feixe Cônico.
\end{abstract}

\section{ABSTRACT}

Aim: The objective of this literature review was to describe studies that evaluated qualitatively and qualitatively the bone mineral density (BMD) using cone beam computed tomography (CBCT) of the maxillomandibular region. This review included articles referring to the use of CBCT in the BMD assessment in the maxillomandibular region from 2009 to 2020, published in the databases: PUBMED, EMBASE and Scopus. The words: "osteoporosis", "bone mineral density" and "bone content" were combined with "CBCT" and "pixel values", using the Boolean operator "AND".

Results: 17 articles were selected. Many researchers used micro-tomography, multislice computed tomography and histomorphometric analysis to make comparisons with the TCFC.

Conclusions: The literature evidences suggest that $\mathrm{CBCT}$, both through its gray scale values and its $3 \mathrm{D}$ structural parameters, presents itself as a method of clinical relevance, reliable and easy, of proportional analysis of the bone density of the maxillomandibular region.

Keywords: Osteoporosis. Bone Density. Cone-Beam Computed Tomography.

\footnotetext{
${ }^{1}$ Departamento de Estomatologia, Universidade de São Paulo.
} 


\section{INTRODUÇÃO}

A osteoporose é uma doença que afeta mais de 200 milhões de pessoas no mundo, causada pelo desequilíbrio entre a reabsorção e a neoformação óssea, que pode estar associado a diversos fatores, resultando em um padrão ósseo com menor densidade mineral e mais suscetível a fratura. ${ }^{1}$ A redução da densidade mineral óssea pode ser averiguada por meio de técnicas imaginológicas de conhecimento do cirurgião-dentista, por exemplo, a tomografia computadorizada de feixe cônico (TCFC).

A TCFC é um método de diagnóstico por imagem da região do complexo maxilomandibular com frequência utilizada na prática clínica, principalmente para as áreas de implantodontia, patologias ósseas e ortodontia, que possibilita a avaliação da estrutura trabecular óssea, qualitativa e quantitativamente. ${ }^{2}$

As tomografias computadorizadas são exames confiáveis no auxílio diagnóstico da qualidade óssea, pois geram imagens em três dimensões com alta resolução, com boa acurácia e mínima distorção. A TCFC possui vantagens ao cirurgião-dentista como reduzido tempo de escaneamento do paciente para obtenção das imagens, baixa dose de radiação e custo, possibilitando maior acessibilidade para a realização desse exame pelo paciente e um maior detalhamento de imagem quando comparado com radiografias panorâmicas e periapicais. ${ }^{3}$

Assim, o objetivo da presente revisão literária foi reportar e discutir estudos que utilizam a TCFC no estudo da densidade mineral óssea (DMO), possibilitando fornecer ao cirurgião-dentista um panorama dos principais estudos qualitativos e quantitativos sobre 0 assunto.

\section{MATERIAIS E MÉTODOS}

\section{Fontes de informação}

Foi realizada uma busca na base de dados busca eletrônica em três bases de dados: Pubmed, Scopus e Embase. A pesquisa foi feita no dia 24 de março de 2020, utilizando combinações das seguintes palavras-chave: "Osteoporosis" AND "CBCT", "bone mineral density" AND "CBCT"; "bone content" AND "CBCT" "pixel values" AND "CBCT" AND "osteoporosis"; "pixel values" AND "CBCT" AND "bone mineral density;" pixel values" AND "CBCT" AND "bone content".

\section{Seleção de estudos}

A seleção de estudos foi realizada da seguinte forma: inicialmente, com a ajuda de um software gerenciador de referência (EndNote ${ }^{\circledR}$ X7 Thomson Reuters, Filadélfia, PA, EUA) utilizado para coletar as referências e excluir as duplicadas. Na sequência, dois revisores revisaram de forma independente os títulos e resumos dos artigos selecionados na busca. Foram excluídos artigos que claramente não se encaixavam nos critérios de inclusão ou que se enquadravam em algum dos critérios de exclusão. Por fim, os artigos remanescentes foram lidos na íntegra e avaliados com base nos mesmos critérios de elegibilidade.

\section{Critério de elegibilidade:}

Para essa revisão foram selecionados artigos de 2009 até 2020 que consideraram métodos de avaliação de densidade mineral óssea maxilomandibular por meio de TCFC.

Foram excluídos trabalhos de revisão, carta, opiniões pessoais, capítulos de livro, relatos de caso, resumos de congressos, estudos realizados fora da região maxilomandibular, estudos nos quais os participantes apresentavam relato de osteonecrose ou osteoradionecrose.

\section{RESULtAdos}

Durante a pesquisa inicial, 1.500 citações foram identificadas em três bases de dados eletrônicas (PubMed, Scopus e Embase). Após a eliminação de trabalhos duplicados, restaram 1.100 artigos. Em seguida, após uma leitura de títulos, 119 artigos tiveram seus resumos lidos. Por serem considerados 
potencialmente úteis, foram selecionados para a avaliação. Dos 119 estudos remanescentes, 43 foram subsequentemente excluídos por não se enquadrarem nos critérios de inclusão, 10 por áreas ou assuntos diferentes ao interesse da revisão e 10 por não avaliarem densidade óssea. Foram lidos na íntegra 56 estudos. Um estudo foi excluído por se tratar de capítulo de livro e mais 41 estudos foram excluídos por serem pôsteres ou revisões da literatura. Foram selecionados 14 artigos e três novas referências foram adicionadas manualmente, oriundas da leitura de referências de artigos selecionados. Portanto, 17 artigos foram incluídos para coleta de dados. A Figura 1 ilustra o processo de identificação e seleção dos estudos. Os artigos selecionados sumarizados encontram-se na Tabela 1.

\section{Artigos localizados $(n=1500)$}

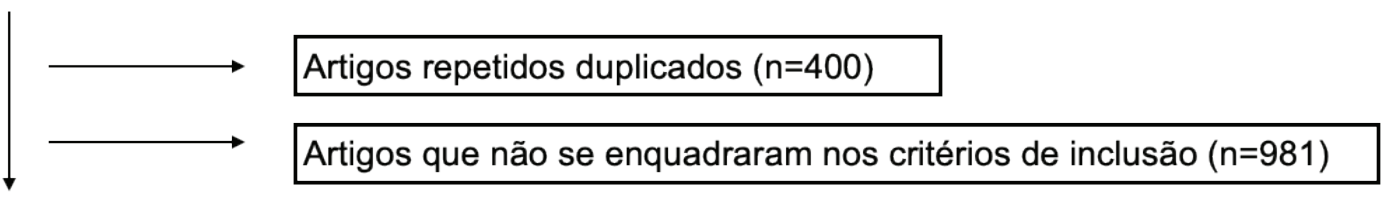

Resumos lidos $(n=119)$

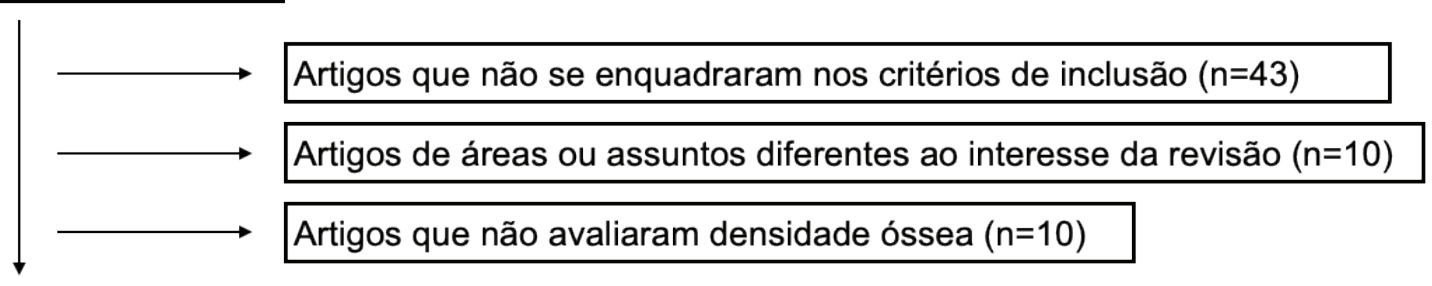

Artigos lidos na íntegra $(n=56)$

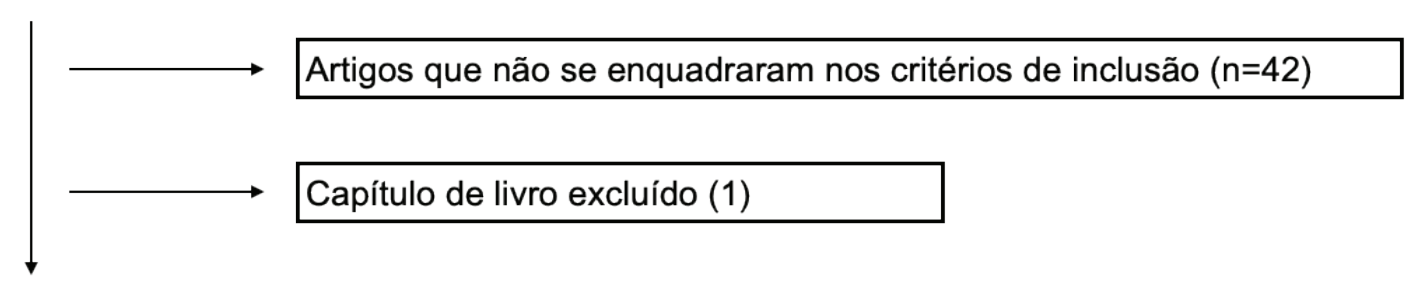

Artigos selecionados $(n=14)$

Artigos incluídos e selecionados manualmente $(n=3)$

Artigos selecionados $(n=17)$

Figura 1: Fluxograma contendo as etapas da revisão de literatura deste estudo. 
BRUNA DI Profio et al.

Tabela 1: Artigos selecionados: autor e ano, objetivo geral do estudo, metodologia, principais resultados e conclusão.

\begin{tabular}{|c|c|c|c|c|}
\hline Autor e ano & Objetivo & Metodologia & Resultados & Conclusão \\
\hline $\begin{array}{l}\text { Hohlweg-Majert et } \\
\text { al., } 2011^{4}\end{array}$ & $\begin{array}{l}\text { Determinar a } \\
\text { quantidade e qualidade } \\
\text { óssea em TCFC em } \\
\text { comparação à TCMS } \\
\text { e } \mu \mathrm{TC} \text {. }\end{array}$ & $\begin{array}{l}\text { Cilindros de osso esponjoso } \\
\text { de mandíbula de amostra } \\
\text { de metades de crânio de } \\
\text { porco. }\end{array}$ & $\begin{array}{l}\text { Há correlação das HU com a } \\
\text { morfologia óssea. É possível } \\
\text { avaliar a DMO usando TCFC. } \\
\text { Os resultados numéricos da } \\
\text { medida da TCFC não são } \\
\text { comparáveis aos resultados } \\
\text { da TCMS. }\end{array}$ & $\begin{array}{l}\text { A TCFC é uma opção adequada para } \\
\text { analisar a massa óssea, mas não } \\
\text { fornece informações sobre a qualidade } \\
\text { óssea com a mesma acurácia da } \mu \text { TC. }\end{array}$ \\
\hline $\begin{array}{l}\text { González-García } \\
\& \text { Monje, } 2013^{5}\end{array}$ & $\begin{array}{l}\text { Analisar a relação } \\
\text { entre a RBD avaliada } \\
\text { pelos valores de } \\
\text { densidade obtidos } \\
\text { pela TCFC e a } \\
\text { microestrutura } \\
\text { histomorfométrica } \\
\text { em } \mu \text { TC dos ossos } \\
\text { maxilares no local dos } \\
\text { implantes dentários }\end{array}$ & $\begin{array}{l}\text { Avaliações em biópsias } \\
\text { ósseas dos ossos maxilares } \\
\text { extraídas de sítios para } \\
\text { instalação de implantes } \\
\text { dentários. }\end{array}$ & $\begin{array}{l}\text { A TCFC é confiável à análise } \\
\text { microestrutural dos ossos } \\
\text { maxilares no momento pré- } \\
\text { operatório. Constatou-se alta } \\
\text { correlação positiva entre RBD } \\
\text { avaliada por TCFC. }\end{array}$ & $\begin{array}{l}\text { A RBD avaliada pela TCFC tem forte } \\
\text { correlação positiva com a BV / TV } \\
\text { avaliada por } \mu \mathrm{TC} \text { no local dos implantes } \\
\text { dentários nos ossos maxilares. }\end{array}$ \\
\hline Taylor et al., $2013^{6}$ & $\begin{array}{l}\text { Examinar a } \\
\text { capacidade da TCFC } \\
\text { no diagnóstico da } \\
\text { diferença relativa do } \\
\text { grau de parâmetros de } \\
\text { mineralização óssea } \\
\text { em uma mandíbula } \\
\text { humana quando } \\
\text { comparada a } \mu \mathrm{TC} .\end{array}$ & $\begin{array}{l}\text { Cortes mandibulares de } \\
\text { cadáveres. } \\
\text { Um histograma do GL, } \\
\text { equivalente ao grau de } \\
\text { mineralização óssea, foi } \\
\text { obtido em cada região das } \\
\text { imagens da TCFC. }\end{array}$ & $\begin{array}{l}\text { As médias das diferenças } \\
\text { percentuais de todos os } \\
\text { parâmetros de GL entre as } \\
\text { regiões de osso medular e } \\
\text { osso cortical basal não foram } \\
\text { significativamente diferentes } \\
\text { entre a } \mu \mathrm{TC} \text { e a TCFC. }\end{array}$ & $\begin{array}{l}\text { A TCFC pode detectar as diferenças } \\
\text { relativas na distribuição mineral do } \\
\text { tecido entre as regiões medulares e } \\
\text { corticais basais no osso da mandíbula, } \\
\text { fornecendo resultados compatíveis aos } \\
\text { da } \mu T C \text {. }\end{array}$ \\
\hline $\begin{array}{l}\text { Van Dessel et al., } \\
2013^{7}\end{array}$ & $\begin{array}{l}\text { Determinar a } \\
\text { precisão da TCFC } \\
\text { para mensurar a } \\
\text { microestrutura do } \\
\text { osso trabecular em } \\
\text { comparação com } \mu \mathrm{CT} \text {. }\end{array}$ & $\begin{array}{l}\text { Amostras de osso humano } \\
\text { mandibular edentulos } \\
\text { foram digitalizados usando } \\
\text { uma máquina TCFC de } \\
\text { alta resolução ( } 80 \mathrm{~mm}) \text {. A } \\
\text { segmentação e morfometria } \\
\text { foram realizadas e } \\
\text { quantificadas dentro dos } \\
\text { volumes de interesse. }\end{array}$ & $\begin{array}{l}\text { Alta correlação entre a TCFC } \\
\text { e } \\
\text { protocolos de } \mu \text { TC para todos } \\
\text { os índices morfométricos } \\
\text { testados, exceto para a } \\
\text { espessura trabecular. }\end{array}$ & $\begin{array}{l}\text { Observou-se um bom potencial da } \\
\text { imagem de alta resolução por TCFC para } \\
\text { aplicações in vivo da morfometria óssea } \\
\text { quantitativa e da avaliação da qualidade } \\
\text { óssea. }\end{array}$ \\
\hline $\begin{array}{l}\text { Soardi et al., } \\
2014^{8}\end{array}$ & $\begin{array}{l}\text { Comparar a TCFC e } \\
\text { a } \mu \text { TC em cirurgias de } \\
\text { levantamento de seio } \\
\text { maxilar, para verificar } \\
\text { o potencial diagnóstico } \\
\text { da TCFC na avaliação } \\
\text { da regeneração óssea. }\end{array}$ & $\begin{array}{l}\text { Pacientes submetidos à } \\
\text { cirurgia de levantamento de } \\
\text { seio maxilar com partículas } \\
\text { de aloenxerto ósseo } \\
\text { TCFC: antes da cirurgia, } \\
\text { após aumento do seio } \\
\text { e imediatamente após a } \\
\text { inserção do implante. }\end{array}$ & $\begin{array}{l}\text { Não se observaram diferenças } \\
\text { significativas na densidade } \\
\text { do tecido ósseo após o } \\
\text { procedimento de levantamento } \\
\text { de seio e instalação de } \\
\text { implante, por meio da TCFC. }\end{array}$ & $\begin{array}{l}\text { O resultado preliminar demonstrou } \\
\text { claramente a previsibilidade da análise } \\
\text { da TCFC. }\end{array}$ \\
\hline
\end{tabular}


MÉTOdOS DE AVALIAÇÃO DE DENSIDADE MINERAL ÓSSEA MAXILOMANDIBULAR POR MEIO DE TOMOGRAFIA CONE BEAM: REVISÃO DE LITERATURA

\begin{tabular}{|c|c|c|c|c|}
\hline Autor e ano & Objetivo & Metodologia & Resultados & Conclusão \\
\hline $\begin{array}{l}\text { Klintstrom et al., } \\
2014^{2}\end{array}$ & $\begin{array}{l}\text { Avaliar como os } \\
\text { parâmetros de imagem } \\
\text { de TCFC odontológico } \\
\text { afeta a precisão na } \\
\text { quantificação de } \\
\text { estruturas ósseas } \\
\text { trabeculares. }\end{array}$ & $\begin{array}{l}\text { Amostras ósseas } \\
\text { (fragmentos de tecido ósseo } \\
\text { do rádio) foram examinadas } \\
\text { utilizando protocolos de } \\
\text { imagem, diferindo em } \\
\text { corrente, voltagem, grau de } \\
\text { rotação, tamanho do voxel, } \\
\text { área de imagem e tempo de } \\
\text { rotação. }\end{array}$ & $\begin{array}{l}\text { Volume ósseo e espessura } \\
\text { trabecular não foram } \\
\text { afetados pelas alterações nos } \\
\text { parâmetros de imagem. } \\
\text { A dose de radiação variou } \\
\text { entre } 269 \text { e } 1153 \text { mGy cm2 }\end{array}$ & $\begin{array}{l}\text { A estrutura óssea trabecular pode ser } \\
\text { quantificada com precisão pela TCFC } \\
\text { odontológica in vitro, e os parâmetros } \\
\text { estruturais obtidos estão fortemente } \\
\text { relacionados aos obtidos pelo } \mu \mathrm{TC} \text {. }\end{array}$ \\
\hline $\begin{array}{l}\text { Cortes et al., } \\
2015^{9}\end{array}$ & $\begin{array}{l}\text { Identificar } \\
\text { características ósseas } \\
\text { quantitativas que } \\
\text { influenciam o torque de } \\
\text { inserção do implante. }\end{array}$ & $\begin{array}{l}\text { Sítios de implantes, } \\
\text { radiografias panorâmicas } \\
\text { e TCFC pré-operatórias } \\
\text { compuseram o } \\
\text { planejamento cirúrgico. } \\
\text { As amostras ósseas } \\
\text { obtidas nas cirurgias } \\
\text { foram avaliadas com DXA } \\
\text { absortometria de raios-X } \\
\text { dupla, } \mu C T \text { e histologia. }\end{array}$ & $\begin{array}{l}\text { Espessura cortical de crista> } \\
0,75 \mathrm{~mm} \text { e espessura normal } \\
\text { do córtex inferior da mandíbula } \\
\text { foram as variáveis mais } \\
\text { significativas. }\end{array}$ & $\begin{array}{l}\text { O córtex inferior da mandíbula é confiável } \\
\text { para a análise da qualidade do tecido } \\
\text { ósseo para pacientes que necessitam } \\
\text { realizar cirurgias de implantes. }\end{array}$ \\
\hline Kim et al., $2015^{10}$ & $\begin{array}{l}\text { Avaliar a viabilidade da } \\
\text { TCFC na avaliação da } \\
\text { microarquitetura óssea } \\
\text { trabecular. }\end{array}$ & $\begin{array}{l}\text { Espécimes de mandíbulas } \\
\text { humanas analisadas } \\
\text { usando } \mu \text { TC e TCFC. A } \\
\text { correlação de parâmetros } \\
\text { tridimensionais entre TCFC } \\
\text { e } \mu \text { TC foi avaliada. }\end{array}$ & $\begin{array}{l}\text { Todos os parâmetros, exceto } \\
\text { a superfície específica } \\
\text { do osso e a espessura } \\
\text { trabecular, apresentaram } \\
\text { correlações positivas. Entre } \\
\text { os parâmetros, o volume } \\
\text { ósseo, o percentual de volume } \\
\text { ósseo, a separação trabecular } \\
\text { e o grau de anisotropia das } \\
\text { imagens de TCFC mostraram } \\
\text { fortes correlações com as das } \\
\text { imagens de } \mu \text { TC. }\end{array}$ & $\begin{array}{l}\text { A maioria dos parâmetros } \\
\text { microarquiteturais da TCFC foi } \\
\text { correlacionada com os da } \mu \text { TC. } \\
\text { Alguns parâmetros microarquiteturais, } \\
\text { especialmente DA, podem ser usados } \\
\text { como fortes preditores da qualidade } \\
\text { óssea na mandíbula humana. }\end{array}$ \\
\hline $\begin{array}{l}\text { Parsa et al., } \\
2015^{11}\end{array}$ & $\begin{array}{l}\text { Analisar a correlação } \\
\text { entre a fração do } \\
\text { volume ósseo (BV / } \\
\text { TV) e as unidades } \\
\text { de Hounsfield da } \\
\text { densidade óssea } \\
\text { em mandíbulas } \\
\text { humanas, por meio } \\
\text { da } \mu \text { TC e TCMS. } \\
\text { Avaliar a precisão da } \\
\text { TCFC na avaliação } \\
\text { da densidade e } \\
\text { microestrutura óssea } \\
\text { trabecular usando } \\
\text { TCMS e } \mu \text { TC como } \\
\text { padrão-ouro de } \\
\text { referência. }\end{array}$ & $\begin{array}{l}\text { Mandíbulas de cadáveres } \\
\text { humanos parcialmente } \\
\text { desdentados. } \\
\text { As varreduras de } \\
\text { TCMS, TCFC e } \mu \text { TC de } \\
\text { cada mandíbula foram } \\
\text { comparadas para selecionar } \\
\text { a região exata de ROI. }\end{array}$ & $\begin{array}{l}\text { Observaram-se fortes } \\
\text { correlações entre a densidade } \\
\text { de TCFC e TCMS e entre as } \\
\text { medidas de TCFC e } \mu \text { TC. } \\
\\
\text { Foi observada excelente } \\
\text { correlação entre TCMS } \\
\text { (expressa em HU) e } \mu \text { TC } \\
\text { (expressa em BV / TV). }\end{array}$ & $\begin{array}{l}\text { Foi constatada correlação positiva entre } \\
\text { volume, qualidade e densidade óssea, } \\
\text { na comparação TCFC e } \mu \text { TC. Isso } \\
\text { sugere que as medições da densidade } \\
\text { óssea poderiam ser usadas para estimar } \\
\text { parâmetros microestruturais ósseos, } \\
\text { evidenciado pela correlação entre GL da } \\
\text { TCFC e BVNT da } \mu T C \text {. }\end{array}$ \\
\hline $\begin{array}{l}\text { Soardi et al., } \\
2016^{3}\end{array}$ & $\begin{array}{l}\text { Analisar a DXA após } \\
\text { cirurgia de enxerto } \\
\text { ósseo alógeno para } \\
\text { elevação de soalho de } \\
\text { seio maxilar através } \\
\text { de TCFC e avaliar os } \\
\text { espécimes obtidos } \\
\text { durante biópsia em } \mu C T \text {. }\end{array}$ & $\begin{array}{l}\text { Pacientes que realizaram } \\
\text { cirurgias de seio maxilar e } \\
\text { biópsias ósseas com TCFC } \\
\text { em momentos diferentes: } \\
\text { antes da cirurgia, no pós- } \\
\text { operatório imediato da } \\
\text { cirurgia, após } 06,10 \text { e } 18 \\
\text { meses respectivamente. }\end{array}$ & $\begin{array}{l}\text { O conteúdo mineral enxertado } \\
\text { desapareceu completamente } \\
\text { entre } 10 \text { e } 11 \text { meses. }\end{array}$ & $\begin{array}{l}\text { TCFC pode ser considerada uma } \\
\text { ferramenta útil para a avaliação óssea } \\
\text { nos casos de cirurgia de reconstruções } \\
\text { ósseas. }\end{array}$ \\
\hline
\end{tabular}


BRUNA DI Profio et al.

\begin{tabular}{|c|c|c|c|c|}
\hline Autor e ano & Objetivo & Metodologia & Resultados & Conclusão \\
\hline $\begin{array}{l}\text { Van Dessel et al., } \\
2016^{12}\end{array}$ & $\begin{array}{l}\text { Comparar a precisão } \\
\text { da TCFC de diferentes } \\
\text { aparelhos em } \\
\text { comparação com a } \\
\text { TCMS e a } \mu \text { TC para } \\
\text { avaliar objetivamente } \\
\text { a qualidade óssea } \\
\text { trabecular e cortical } \\
\text { antes da colocação de } \\
\text { implantes. }\end{array}$ & $\begin{array}{l}\text { Mandíbulas humanas } \\
\text { tomografadas em } 7 \\
\text { diferentes aparelhos de } \\
\text { TCFC, } 1 \text { aparelho de TCMS, } \\
\text { com duas resoluções no } \\
\text { aparelho (alta resolução } \\
\text { e resolução padrão) e } 1 \\
\text { aparelho para } \mu \mathrm{TC} \text {. }\end{array}$ & $\begin{array}{l}\text { Fração de volume trabecular, } \\
\text { espessura trabecular e fração } \\
\text { de volume cortical foram } \\
\text { superestimados em todas } \\
\text { as máquinas TCMS e TCFC } \\
\text { comparados aos valores } \\
\text { obtidos a partir da } \mu \text { TC. }\end{array}$ & $\begin{array}{l}\text { TCFC de baixa dose possui potencial } \\
\text { similar ao da } \mu \text { TC para a avaliação } \\
\text { estrutural 3D quantitativa do osso } \\
\text { cortical e trabecular antes da instalação } \\
\text { de implantes. Entretanto, nem todas } \\
\text { as máquinas de TCFC são igualmente } \\
\text { confiáveis na exibição da estrutura do } \\
\text { osso trabecular e cortical. A TCMS de } \\
\text { resolução padrão deixa a desejar a esse } \\
\text { respeito. }\end{array}$ \\
\hline $\begin{array}{l}\text { Van Dessel et al., } \\
2017^{13}\end{array}$ & $\begin{array}{l}\text { Verificar clinicamente } \\
\text { se a TCFC pode } \\
\text { ser usada de forma } \\
\text { confiável na avaliação } \\
\text { da qualidade } \\
\text { óssea alveolar em } \\
\text { comparação com } \\
\text { a TCMS e o a } \mu T C \\
\text { (padrão-ouro). }\end{array}$ & $\begin{array}{l}\text { Amostras de mandíbulas } \\
\text { dentadas de cadáveres }\end{array}$ & $\begin{array}{l}\text { Os índices de TCFC e } \\
\text { TCMS mostraram uma rede } \\
\text { de trabéculas alveolares } \\
\text { significativamente mais } \\
\text { espessas, associado } \\
\text { a uma superestimação } \\
\text { significativa do volume ósseo } \\
\text { e poros mais próximos em } \\
\text { comparação a } \mu \mathrm{CT} \text {. }\end{array}$ & $\begin{array}{l}\text { As medidas morfométricas obtidas pela } \\
\text { maioria das máquinas TCFC e pelas } \\
\text { TCMS de alta resolução são confiáveis, } \\
\text { apesar de superestimarem a quantidade } \\
\text { de osso em comparação à } \mu \text { TC. A } \\
\text { análise do padrão estrutural do osso } \\
\text { alveolar, permanece semelhante para as } \\
\text { máquinas TCFC de mais alta resolução. }\end{array}$ \\
\hline $\begin{array}{l}\text { Wang et al., } \\
2017^{14}\end{array}$ & $\begin{array}{l}\text { Verificar a } \\
\text { confiabilidade da } \\
\text { TCFC na avaliação } \\
\text { da densidade óssea } \\
\text { através da análise da } \\
\text { escala de contraste, } \\
\text { comparando-a com os } \\
\text { dados obtidos na } \mu \mathrm{CT} \text {. }\end{array}$ & $\begin{array}{l}\text { Biópsias ósseas e análises } \\
\text { morfométricas realizadas } \\
\text { com as imagens obtidas em } \\
\text { amostras selecionadas de } \\
\text { osso mandibular. }\end{array}$ & $\begin{array}{l}\text { Das amostras, } 80 \% \text { tiveram } \\
\text { os valores entre } 350 \text { e } 550 \text { na } \\
\text { escala de contraste das TCFC. } \\
\text { Foi encontrada correlação } \\
\text { diretamente proporcional } \\
\text { para a densidade média e } \\
\text { correlação inversamente } \\
\text { proporcional para o espaço } \\
\text { trabecular. }\end{array}$ & $\begin{array}{l}\text { Existe correlação entre o padrão do } \\
\text { tecido ósseo avaliado por TCFC e a } \\
\text { densidade óssea analisada por } \mu \mathrm{TC} \text { na } \\
\text { região posterior de mandíbula. }\end{array}$ \\
\hline $\begin{array}{l}\text { Nishimura et al., } \\
2018^{15}\end{array}$ & $\begin{array}{l}\text { Correlacionar e } \\
\text { comparar os valores } \\
\text { em pixel através } \\
\text { de TCFC em seios } \\
\text { maxilares enxertados } \\
\text { com estruturaras } \\
\text { ósseas adjacentes. } \\
\end{array}$ & $\begin{array}{l}\text { TCFC em pacientes que } \\
\text { passaram por cirurgia de } \\
\text { elevação de seio maxila } \\
\text { com enxerto de fosfato de } \\
\text { cálcio bifásico. }\end{array}$ & $\begin{array}{l}\text { Correlações positivas foram } \\
\text { encontradas entre a área } \\
\text { enxertada e a área óssea } \\
\text { adjacente }\end{array}$ & $\begin{array}{l}\text { Os valores em pixel de seios maxilares } \\
\text { enxertados estão diretamente associados } \\
\text { à extensão da área enxertada. }\end{array}$ \\
\hline $\begin{array}{l}\text { Tanaka et al., } \\
2018^{16}\end{array}$ & $\begin{array}{l}\text { Avaliar a } \\
\text { microestrutura do } \\
\text { trabeculado ósseo } \\
\text { após exodontias. }\end{array}$ & $\begin{array}{l}\text { Biópsias ósseas foram } \\
\text { avaliadas de alvéolos pós- } \\
\text { exodontias através de } \mu \mathrm{CT} \\
\text { e análise histológica. }\end{array}$ & $\begin{array}{l}\text { No período entre o } 7^{\circ} \text {. e o } \\
12^{\circ} \text {. mês pós exodontia há } \\
\text { atividades de remodelação } \\
\text { óssea no alvéolo e após o } 12^{\circ} \text {. } \\
\text { mês ocorreu uma estabilidade } \\
\text { no processo de maturação } \\
\text { óssea. }\end{array}$ & $\begin{array}{l}\text { A remodelação do trabeculado ósseo } \\
\text { em humanos após exodontias possui um } \\
\text { período de estabilização. }\end{array}$ \\
\hline $\begin{array}{l}\text { Brehler et al., } \\
2018^{17}\end{array}$ & $\begin{array}{l}\text { Investigar o } \\
\text { desempenho da TCFC } \\
\text { na análise quantitativa } \\
\text { do osso trabecular. }\end{array}$ & $\begin{array}{l}\text { Biópsias ósseas de } \\
\text { pacientes com tolerância } \\
\text { prejudicada à glicose e com } \\
\text { diabetes mellitus tipo } 2 \text {. }\end{array}$ & $\begin{array}{l}\text { De acordo com as medições } \\
\text { da } \mu \text { TC, as } 35 \text { amostras } \\
\text { transiliacas cobriram uma } \\
\text { ampla faixa de valores de BV / } \\
\text { TV, de } 7,5 \% \text { a } 50,5 \% \text {; } \\
\text { A comparação à segmentação } \\
\text { global da linha de base } \\
\text { usando o método de Otsu } \\
\text { melhorou a correlação TCFC } \\
\text { e } \mu \text { TC; a melhora foi de: } \\
22 \% \text { para BV / TV, 27\% para } \\
\text { espessura do trabeculado, } \\
56 \% \text { para espaço trabecular e } \\
\sim 31 \% \text { para número trabecular. }\end{array}$ & $\begin{array}{l}\text { A TCFC pode ter utilidade na detecção } \\
\text { de alterações na microestrutura óssea } \\
\text { induzida por doença ou intervenção } \\
\text { terapêutica. }\end{array}$ \\
\hline
\end{tabular}


MÉTODOS DE AVALIAÇÃO DE DENSIDADE MINERAL ÓSSEA MAXILOMANDIBULAR POR MEIO DE TOMOGRAFIA CONE BEAM: REVISÃo DE LITERATURA

\begin{tabular}{|c|c|c|c|c|}
\hline Autor e ano & Objetivo & Metodologia & Resultados & Conclusão \\
\hline $\begin{array}{l}\text { Kulah et al., } \\
2019^{18}\end{array}$ & $\begin{array}{l}\text { Avaliar a } \\
\text { microarquitetura } \\
\text { trabecular da maxila } \\
\text { usando } 2 \text { imagens } \\
\text { de TCFC diferentes, } \\
\text { obtidas com diferentes } \\
\text { configurações em } \\
\text { comparação com } \\
\text { imagens de } \mu \mathrm{TC} \text { ex } \\
\text { vivo. }\end{array}$ & $\begin{array}{l}\text { Aparelhos: (TCFC) 3-D } \\
\text { Planmeca Promax Max e } \\
\text { 3-D Accuitomo 170; }(\mu C T) \text { : } \\
\text { SkyScan 1.174. } \\
\text { Foram avaliadas } 17 \\
\text { amostras de maxilas } \\
\text { posteriores de cadáveres } \\
\text { por } 2 \text { tipos de aparelhos } \\
\text { diferentes de TCFC e por } \\
\text { um } \mu \text { TC. }\end{array}$ & $\begin{array}{l}\text { A relação BV / TV e os } \\
\text { parâmetros DA mostraram } \\
\text { concordância significativa } \\
\text { entre dispositivos de } \\
\mu C T \text { e TCFC. Imagens } \\
\text { de TCFC obtidas com o } \\
\text { menor tamanho de voxel } \\
\text { fornecem informações mais } \\
\text { precisas para a avaliação da } \\
\text { microestrutura óssea maxilar. }\end{array}$ & $\begin{array}{l}\text { A relação BV / TV e os parâmetros DA } \\
\text { medidos na TCFC obtida no menor } \\
\text { tamanho de voxel, mostrou-se útil para } \\
\text { a avaliação da microestrutura trabecular } \\
\text { da maxila. }\end{array}$ \\
\hline
\end{tabular}

Abreviações: TCMS tomografia computadorizada multislice; TCFC tomografia computadorizada de feixe cônico; $\mu$ TC micro tomografia computadorizada; HU unidades de Hounsfield; RBD radiographic bone density (densidade radiográfica óssea); BV bone volume; TV tissue; BV/TV fração óssea volumétrica; GL gray level (escala de cinza); ROI region of interest (região de interesse); DMO: densidade mineral óssea; DXA: densitometria óssea; GSD: grayscale density (densidade da escala de cinza).

Dezessete artigos preencheram os critérios de inclusão e exclusão. ${ }^{2-18}$ Os artigos avaliaram assuntos diversos, com comparações da TCFC à análises de microtomografia $(\mu \mathrm{CT})$; análises histológicas; comparações entre TCFC e TCMS; comparação entre aparelhos de TCFC; e a influência dos parâmetros de imagem na análise da densidade mineral óssea.

\section{Discussão}

A presente revisão da literatura sumarizou os estudos disponíveis sobre a confiabilidade diagnóstica da TCFC na avaliação da densidade mineral óssea maxilomandibular. Para tanto, entre uma vasta quantidade de trabalhos encontrados na literatura, 17 artigos foram utilizados, ${ }^{2-18}$ nos quais o exame de TCFC foi comparado de diversas formas com outras técnicas de imagem ou mesmo análises histomorfométricas.

A TCFC é um exame atualmente muito utilizado na Odontologia devido ao baixo custo, baixa dose de radiação e maior rapidez de realização quando comparado à TCMS. Porém, sua eficácia e confiabilidade na avaliação da densidade óssea na região de maxila e mandíbula ainda é um assunto controverso, ${ }^{9,14}$ uma vez que os sistemas de TCFC possuem a desvantagem, quando comparados às TCMS, de ausência correlação da intensidade de pixel provida pela TCFC com as unidades Hounsfield (HU), ${ }^{18}$ que possuem comprovada correlação com a densidade mineral do tecido ósseo, ${ }^{19-21}$ embora as unidades HU não devam ser usadas para fins de diagnóstico da redução da DMO.

A osteoporose é uma desordem no processo de redução da densidade mineral óssea (DMO) e deterioração microestrutural do tecido ósseo, levando ao enfraquecimento ósseo e consequentes fratura. ${ }^{22} \mathrm{~A}$ osteoporose apresenta forte associação idade, menopausa precoce, deficiência de vitamina D, hipertireoidismo, hiperparatireoidismo, diabetes mellitus tipo 1, doença de Cushing, sedentarismo, etnia (caucasiana), histórico familiar de fraturas, hábitos tóxicos tabagismo, etilismo, abuso de drogas e medicamentos, principalmente o uso de corticosteroides, baixa ingestão de cálcio (ainda que controverso) e desnutrição. Por se tratar de uma desordem que não apresenta sintomatologia dolorosa, é considerada "a epidemia silenciosa". A morbidade decorre da complexidade e da importância das fraturas. ${ }^{23} \mathrm{~A}$ alteração da 
densidade mineral óssea (DMO) se manifesta tanto sistemicamente, quanto no complexo maxilo-mandibular. ${ }^{24}$

As avaliações da DMO feitas através das TCFCsãorealizadasporintermédiodesoftwares que, assim como nas TCMS, se baseiam na associação direta com os coeficientes de atenuação dos tecidos, onde os valores de pixels são mensurados, apresentando valores diretamente proporcionais à quantidade de mineralização do tecido. Os valores de pixels são correlacionados à escala de contraste formada pelos diversos tons de cinza da imagem tomográfica em questão (análise 2D), sendo que a extensão da escala de contraste da imagem depende da quantidade de bit do aparelho que a realizou.

Hohlweg-Majert et al. (2011) ${ }^{4}$ e Parsa et al. $(2015)^{11}$ demonstraram, que apesar dos resultados numéricos dos valores de pixels das TCFC não terem correlação com os valores de HU obtidos nas TCMS, a escala de contraste de uma imagem de TCFC é ferramenta capaz de aferir a densidade mineral de um tecido ósseo. ${ }^{6} \mathrm{~A}$ análise quantitativa dos valores de pixel mensurados na imagem em TCFC pode ser útil no rastreamento de pacientes sob risco de redução da DMO.

Para a verificação da eficácia da TCFC na avaliação da densidade óssea maxilomandibular, estudos têm utilizado como padrão ouro de referência, dados de amostras do mesmo indivíduo obtidos por meio da $\mu \mathrm{TC}$, que é um exame que não possui aplicação clínica, mas que, pela comparação, pode validar o uso clínico da TCFC para esse fim. ${ }^{5}$, $6,8-12,15$

Reforçando a validação da TCFC como ferramenta de análise da DMO, por meio da $\mu \mathrm{TC}$, podemos citar os trabalhos de GozalezGarcia et al. (2013), ${ }^{5}$ Soardi et al. $(2014)^{8}$ e $(2016)^{3}$ e Parsa et al. (2015), ${ }^{11}$ que mostraram forte relação positiva entre o volume do tecido ósseo obtido por meio da $\mu \mathrm{TC}$ com as medidas da densidade óssea radiográfica obtidas através da TCFC. No entanto, Kim et al. $(2015)^{10}$ mencionaram que é necessário levar em consideração a possibilidade de que alguns parâmetros morfométricos da $\mu \mathrm{TC}$ podem apresentar valores aumentados quando avaliados em imagens de reconstrução 3D de TCFC e, dessa forma, medições precisas devem ser aprimoradas, diminuindo dessa maneira o tamanho do voxel e a quantidade de ruído dessas tomografias.

Van Dessel et al. (2016)12 demonstraram o potencial da TCFC em baixa dose na avaliação estrutural 3D quantitativa do osso cortical e trabecular, mas salientaram que nem todos os equipamentos de TCFC e TCMS de resolução padrão são igualmente confiáveis na exibição da estrutura trabecular e cortical do osso, o que é corroborado pelos estudos de HohlwegMajert et al. (2011). ${ }^{4}$

Cortes et al. $(2015)^{9}$ avaliaram imagens de radiografias panorâmicas e de TCFC préoperatórias para os implantes dentários, escaneamentos de $\mu$-TC, exames histológicos e análises de densitometrias ósseas dessas mesmas amostras, e compararam esses achados com as características ósseas quantitativas que influenciam $o$ torque de inserção dos implantes, e os achados observados, nesse trabalho, sugerem que as varreduras de TCFC realizadas podem ser consideradas para avaliar a densidade óssea alveolar, pois a densidade óssea radiográfica obtida com esse método estava fortemente correlacionada com as medidas morfométricas de ambos $\mu \mathrm{TC}$ e histologia.

Taylor et al. $(2013)^{6}$ encontraram uma distribuição significativa do nível do contraste de cinza, que são equivalentes aos parâmetros do grau de mineralização óssea, e esse achado sugere que as avaliações para os valores relativos do grau de mineralização óssea com base nas imagens obtidas por meio da TCFC são comparáveis àqueles baseados na $\mu \mathrm{TC}$ de 
MÉTOdOS DE AVALIAÇÃo DE DENSIDADE MINERAL ÓSSEA MAXILOMANDIBULAR POR MEIO DE TOMOGRAFIA CONE BEAM: REVISÃO DE LITERATURA

alta resolução. Hohlweg-Majert et al. (2011) ${ }^{4}$ concordam que a densidade mineral óssea pode ser avaliada usando imagens de uma TCFC de uso clínico, mas que para a obtenção de informações relativas à força e estabilidade óssea, deve ser feita uma reconstrução 3D, e o volume de interesse deve ser então analisado.

Além da relevância clínica discutida sobre a avaliação da densidade do osso nativo na região maxilomandibular por meio da TCFC, Soardi et al. $(2014)^{8}$ e $(2016)^{3}$ e Nishimura et al. (2018), ${ }^{15}$ avaliando amostras ósseas removidas de áreas enxertadas, concluíram que os valores de pixel da TCFC foram descritos como adequados para avaliar indiretamente a densidade óssea, pois fortes correlações foram encontradas entre os valores de pixels e os achados histomorfométricos.

\section{CONSIDERAÇÕES FINAIS}

Levando-se em conta os achados obtidos nesta revisão literária, podemos concluir que as evidências bibliográficas sugerem que a TCFC, tanto através dos seus valores de escala de cinza quanto pelos seus parâmetros 3D estruturais, se apresenta como um método de relevância clínica confiável e de fácil acesso, de análise proporcional da densidade óssea da região maxilomandibular.

\section{REFERÊNCIAS}

1. Munhoz L., Aoki EM, Cortes ARG, de Freitas $\mathrm{CF}$, Arita ES. Osteoporotic alterations in a group of different ethnicity Brazilian postmenopausal women: An observational study. Gerodontology. 2018, jun.; 35: 101-9.

https://pubmed.ncbi.nlm.nih gov/29380906/?from_single_result=Osteoporot ic+alterations+in+a+group+of+different+ethnici ty+Brazilian+postmenopausal+women $\% 3 A+A n$ +observational+study\&expanded_search_quer $y=$ Osteoporotic+alterations+in+a+group+of+diff erent+ethnicity+Brazilian+postmenopausal+wo men $\% 3 A+A n+o b s e r v a t i o n a l+s t u d y$
2. Klintström E., Smedby O., Klintström B., Brismar TB, Moreno R. Trabecular bone histomorphometric measurements and contrast-to-noise ratio in CBCT. Dentomaxillofac Radiol. 2014, aug.; 43: 20140196.

h t t p s: // pubmed.ncbi.n Im.nih. gov/25168811/?from_single_result=Trabecula $\mathrm{r}+$ bone+histomorphometric+measurements+a nd+contrast-to-noise+ratio+in+CBCT\&expanded_search_query=Trabecular+bone+histomor phometric+measurements+and+contrast-to-noi se+ratio+in+CBCT

3. Soardi CM, Suárez-López del Amo F., Galindo-Moreno P., Catena A., Zaffe D., Wang HL. Reliability of Cone Beam Computed Tomography in Determining Mineralized Tissue in Augmented Sinuses. Int J Oral Maxillofac Implants. 2016, mar./apr.; 31: 352-8.

https://pubmed.ncbi.nlm.nih.gov/27004281/

4. Hohlweg-Majert B., Metzger MC, Kummer T., Schulze D. Morphometric analysis - Cone beam computed tomography to predict bone quality and quantity. J Craniomaxillofac Surg. 2011, jul.; 39: 330-4.

ht tps://pubmed.ncbi.nIm.nih. gov/21030266/? from_single result=Morphometric+analysis+-+Cone+bea $\mathrm{m}+$ computed+tomography+to+predict+bone +quality+and+quantity\&expanded_search_ query=Morphometric+analysis+-+Cone+beam+ computed+tomography+to+predict+bone+quali ty+and+quantity

5. González-García R., Monje F. The reliability of cone-beam computed tomography to assess bone density at dental implant recipient sites: a histomorphometric analysis by micro-CT. Clin Oral Implants Res. 2013, aug.; 24: 871-879. https://pubmed. ncbi.nlm.nih.gov/22250839/?from_single_ result=he+reliability+of+cone-beam+computed +tomography+to+assess+bone+density+at+de ntal+implant+recipient+sites $\% 3 \mathrm{~A}+\mathrm{a}+$ histomorp hometric+analysis+by+micro-CT.\&expanded_ search_query=he+reliability+of+cone-beam + co mputed+tomography+to+assess+bone+density +at+dental+implant+recipient+sites $\% 3 \mathrm{~A}+\mathrm{a}+$ hist omorphometric+analysis+by+micro-CT. 
BRUNA DI PRofio et al.

6. Taylor TT, Gans SI, Jones EM, Firestone AR, Johnston WM, Kim DG. Comparison of microCT and cone beam CT-based assessments for relative difference of grey level distribution in a human mandible. Dentomaxillofac Radiol. 2013, sep.; 42: 25117764. https:// pubmed.ncbi.nlm.nih.gov/22996393/?from_ single_result $=$ Comparison + of + micro $\mathrm{CT}+$ and +cone+beam+CT-based+assessme nts+for+relative+difference+of+grey+level+ distribution+in+a+human+mandible.\&expan ded_search_query=Comparison+of+micro$\mathrm{CT}+$ and+cone+beam+CT-based+assessments + for+relative+difference+of+grey+level+distribu tion+in+a+human+mandible.

7. Van Dessel J., Huang Y., Depypere M., Rubira-Bullen I., Maes F., Jacobs R. A comparative evaluation of cone beam CT and micro-CT on trabecular bone structures in the human mandible. Dentomaxillofac Radiol. 2013, jul.; 42: 20130145. https://pubmed.ncbi.nlm.nih. gov/23833320/

8. Soardi CM, Zaffe D., Motroni A., Wang HL. Quantitative comparison of cone beam computed tomography and microradiography in the evaluation of bone density after maxillary sinus augmentation: a preliminary study. Clin Implant Dent Relat Res. 2014, aug.; 16: 557-64.

h t t p s: // pub med.ncbi.n Im.nih. gov/23157713/?from_single_result=Quantitat ive+comparison+of+cone+beam+computed $+t$ omography+and+microradiography+in+the+e valuation+of+bone+density+after+maxillary+s inus+augmentation $\% 3 \mathrm{~A}+\mathrm{a}+$ preliminary+study \&expanded_search_query=Quantitative+com parison+of+cone+beam+computed+tomograp hy+and+microradiography+in+the+evaluation +of+bone+density+after+maxillary+sinus+aug mentation $\% 3 \mathrm{~A}+\mathrm{a}+$ preliminary+study

9. Cortes AR, Eimar H., Barbosa JeS, Costa C., Arita ES, Tamimi F. Sensitivity and specificity of radiographic methods for predicting insertion torque of dental implants. J Periodontol. 2015, jan.; 86: 646-65. https://pubmed.ncbi.nlm.nih. gov/25594426/?from_term=Sensitivity+and+sp ecificity+of+radiographic+methods+for+predicti ng+insertion+torque+of+dental+\&from_pos=1
10. Kim JE, Yi WJ, Heo MS, Lee SS, Choi SC, Huh KH. Three-dimensional evaluation of human jaw bone microarchitecture: correlation between the microarchitectural parameters of cone beam computed tomography and micro-computer tomography. Oral Surg Oral Med Oral Pathol Oral Radiol. 2015, dec.; 120: 762-70. https://pubmed.ncbi.nlm.nih. gov/26548728/?from_single_result=Three-di mensional+evaluation+of+human+jaw+bone + microarchitecture $\% 3 \mathrm{~A}+$ correlation+between + the+microarchitectural+parameters + of + con e+beam+computed+tomography+and+microcomputer+tomography. \&expanded_. search_query=Three-dimensional+evalu ation+of+human+jaw+bone+microarchite cture $\% 3 \mathrm{~A}+$ correlation+between+the + mic roarchitectural+parameters+of+cone +be am+computed+tomography+and+microcomputer+tomography.

11. Parsa A., Ibrahim N., Hassan B., van der Stelt P., Wismeijer D. Bone quality evaluation at dental implant site using multislice CT, micro$\mathrm{CT}$, and cone beam CT. Clin Oral Implants Res. 2015, dec.; 26: e1-7. https://pubmed. ncbi.nlm.nih.gov/24325572/?from_single_ result $=$ Bone + quality + evaluation + at + dental $+\mathrm{i}$ mplant+site+using+multislice $+\mathrm{CT} \% 2 \mathrm{C}+$ micro $-\mathrm{CT} \% 2 \mathrm{C}+$ and+cone+beam+CT.\&expanded_ search_query=Bone+quality+evaluation+at+de ntal+implant+site+using+multislice $+\mathrm{CT} \% 2 \mathrm{C}+\mathrm{mi}$ cro-CT\%2C+and+cone+beam+CT.

12. Van Dessel J., Nicolielo LF, Huang Y., et al. Quantification of bone quality using different cone beam computed tomography devices: Accuracy assessment for edentulous human mandibles. Eur J Oral Implantol. 2016; 9: 411-24.

h t t p s: // pubmed.ncbi.nIm.nih. gov/27990508/?from_single_result=Quantificati on+of+bone+quality+using+different+cone+bea $\mathrm{m}+$ computed+tomography+devices $\% 3 \mathrm{~A}+\mathrm{Accu}$ racy+assessment+for+edentulous+human+ma ndibles.\&expanded_search_query=Quantificati on+of+bone+quality+using+different+cone+bea $\mathrm{m}+$ computed+tomography+devices $\% 3 \mathrm{~A}+\mathrm{Accur}$ acy+assessment+for+edentulous+human+man dibles. 
MÉTOdOS DE AVALIAÇÃo DE DENSIDADE MINERAL ÓSSEA MAXILOMANDIBULAR POR MEIO DE TOMOGRAFIA CONE BEAM: REVISÃO DE LITERATURA

13. Van Dessel J., Nicolielo LF, Huang Y., et al. Accuracy and reliability of different cone beam computed tomography (CBCT) devices for structural analysis of alveolar bone in comparison with multislice CT and micro-CT. Eur J Oral Implantol. 2017; 10: 95-105.

h t t p s: // pubmed.ncbi.nIm.nih. gov/28327698/?from_single_result=ccuracy+ and+reliability+of+different+cone+beam+com puted+tomography+\%28CBCT\%29+devices+ for+structural+analysis+of+alveolar+bone+in+ comparison+with+multislice+CT+and+micro-CT\&expanded_search_query=ccuracy + and $+r$ eliability+of+different+cone+beam+computed+ tomography+\%28CBCT\%29+devices+for+stru ctural+analysis+of+alveolar+bone+in+compari son+with+multislice+CT+and+micro-CT

14. Wang F., Huang W., Wu Y., et al. Accuracy of Cone Beam Computed Tomography Grayscale Density in Determining Bone Architecture in the Posterior Mandible: An In Vivo Study with Microcomputed Tomography Validation. Int J Oral Maxillofac Implants. 2017, apr.; 32: 1.074-79. https://pubmed.ncbi.nlm.nih. gov/28403249/?from_single_result=Accuracy+ of+Cone+Beam+Computed+Tomography+Gra yscale+Density+in+Determining+Bone+Archite cture+in+the+Posterior+Mandible\%3A+An+In+ Vivo+Study+with+Microcomputed+Tomography +Validation\&expanded_search_query=Accurac $y+o f+C o n e+B e a m+C o m p u t e d+T o m o g r a p h y+G$ rayscale+Density+in+Determining+Bone+Archi tecture+in+the+Posterior+Mandible $\% 3 A+A n+l n$ +Vivo+Study+with+Microcomputed+Tomograp hy+Validation

15. Nishimura DA, Aoki EM, Abdala Júnior R., et al. Comparison of Pixel Values of Maxillary Sinus Grafts and Adjacent Native Bone With ConeBeam Computed Tomography. Implant Dent. 2018, dec.; 27: 667-71. https://pubmed.ncbi.nIm. nih.gov/30320616/?from_single_result=Compari son+of+Pixel+Values+of+Maxillary+Sinus+Graft s+and+Adjacent+Native+Bone+With+Cone-Bea $\mathrm{m}+$ Computed+Tomography.\&expanded_search_ query=Comparisn+of+Pixel+Values+of+Maxillary + Sinus+Grafts+and+Adjacent+Native+Bone+Wit $\mathrm{h}+$ Cone-Beam+Computed+Tomography.
16. Tanaka M., Yamashita-Mikami E., Akazawa K., Yoshizawa M., Arai Y., Ejiri S. Trabecular bone microstructure and mineral density in human residual ridge at various intervals over a long period after tooth extraction. Clin Implant Dent Relat Res. 2018, jun.; 20: 375-83. https:// pubmed.ncbi.nlm.nih.gov/29508527/?from_single_result=Trabecular+bone+microstructure $+a$ nd+mineral+density+in+human+residual+ridge +at+various+intervals+over+a+long+period+aft er+tooth+extraction.\&expanded_search_query $=$ Trabecular+bone+microstructure+and+minera I+density+in+human+residual+ridge+at+variou s+intervals+over+a+long+period+after+tooth+e xtraction.

17. Brehler M., Cao Q., Moseley KF, et al. Robust Quantitative Assessment of Trabecular Microarchitecture in Extremity Cone-Beam CT Using Optimized Segmentation Algorithms. Proc SPIE Int Soc Opt Eng. 2018, mar.; 10578. https:// pubmed.ncbi.nlm.nih.gov/31337926/?from_single_result=Robust+Quantitative+Assessmen $\mathrm{t}+\mathrm{of}+$ Trabecular+Microarchitecture $+\mathrm{in}+$ Extre mity+Cone-Beam+CT+Using+Optimized+Se gmentation+Algorithms.\&expanded_search_ query=Robust+Quantitative+Assessment+of $+\bar{T}$ rabecular+Microarchitecture+in+Extremity+Con e-Beam+CT+Using+Optimized+Segmentation + Algorithms.

j18. Kulah K., Gulsahi A., Kamburoğlu K., et al. Evaluation of maxillary trabecular microstructure as an indicator of implant stability by using 2 cone beam computed tomography systems and micro-computed tomography. Oral Surg Oral Med Oral Pathol Oral Radiol. 2019, mar.; 127: 247-56. https://pubmed. ncbi.nlm.nih.gov/30595501/?from_single_re sult $=$ Evaluation + of + maxillary+trabecular+ microstructure+as+an+indicator+of+impla $n t+$ stability+by+using $+2+$ cone + beam + com puted+tomography+systems+and+microcomputed+tomography.\&expanded_search_ query=Evaluation+of+maxillary+trabecular + microstructure+as+an+indicator+of $+i m p l$ ant + stability+by+using $+2+$ cone + beam + co mputed+tomography+systems+and+microcomputed+tomography. 
19. Munhoz L., Takahashi DN, Ramos EAA, Tenorio JR, Arita ES. Do patients with osteoporosis have higher risk to present reduced alveolar ridge height? An Imaging Analysis. Indian J Dent Res. 2019, sep./oct.; 747-50.

https://pubmed.ncbi.nlm. nih.gov/31854367/?from term=osteoporosis+munhoz\&from_pos=1

20. Zhang Q., Jing D., Zhang Y., Miron RJ. Histomorphometric Study of New Bone Formation Comparing Defect Healing with Three Bone Grafting Materials: The Effect of Osteoporosis on Graft Consolidation. Int J Oral Maxillofac Implants. 2018, may/jun.; 33: 645-52. https:// pubmed.ncbi.nlm.nih.gov/29420674/?from_ single_result $=$ Histomorphometric + Study + of +New+Bone+Formation+Comparing+Defec $\mathrm{t}+$ Healing +with+Three+Bone+Grafting +Mat erials $\% 3 A+T h e+E f f e c t+o f+O s t e o p o r o s i s+o n-$ + Graft+Consolidation\&expanded_search_quer $y=$ Histomorphometric + Study + of + New + Bone $+F$ ormation+Comparing+Defect+Healing+with+Th ree+Bone+Grafting+Materials\%3A+The+Effect +of+Osteoporosis+on+Graft+Consolidation

21. Morita L., da Silva VB, Munhoz L., Arita ES, Watanabe PCA. Evaluation and Correlation be- tween craniofacial bone densities and C1 and C2 cervical vertebrae using multislice computed tomography. Brazilian Dental Science. 2019, oct./dec.: 546-53.

https://doi.org/10.14295/bds.2019.v22i4.1839

22. Munhoz L. Cl, Miura DK, Watanabe PCA, Arita ES. Bone mineral density and mandibular osteoporotic alterations in panoramic radiographs: Correlation by peripheral bone densitometry in men. Indian J Den Res. 2019 [epub ahead of print] https://doi.org/10.4103/ijdr.IJDR_440_18

23. Choi I ML, Arita ES. Assessment of Osteoporotic Alterations in Brazilian Postmenopausal Women: A Retrospective Study. Journal of Clinical and Dental Research. 2018, may; 12: ZC34-ZC37. https://doi.org/10.7860/JCDR/2018/34529

24. Horner K., Devlin H., Alsop CW, Hodgkinson IM, Adams JE. Mandibular bone mineral density as a predictor of skeletal osteoporosis. Br J Radiol. 1996, nov.; 69: 1.019-25.

https://pubmed.ncbi.nlm.nih.gov/8958019/

Submetido em: $24-5-2020$

Aceito em: 3-9-2020 